\title{
Cerebral asymmetries in sleep-dependent processes of memory consolidation
}

\author{
Philippe Peigneux, ${ }^{1,3,4,5}$ Remy Schmitz, ${ }^{1,3}$ and Sylvie Willems ${ }^{2,3}$ \\ ${ }^{1}$ Cyclotron Research Centre, University of Liège, B-4000 Liège, Belgium; ${ }^{2}$ Neuropsychology Unit at the Department of Cognitive \\ Sciences, University of Liège, B-4000 Liège, Belgium
}

\begin{abstract}
Preference for previously seen, unfamiliar objects reflects a memory bias on affective judgment, known as the "mere exposure effect" (MEE). Here, we investigated the effect of time, post-exposure sleep, and the brain hemisphere solicited on preference generalization toward objects viewed in different perspectives. When presented in the right visual field (RVF), which promotes preferential processing in the left hemisphere, same and mirrored exemplars were preferred immediately after exposure. MEE generalized to much dissimilar views after three nights of sleep. Conversely, object presentation in the left visual field (LVF), promoting right hemisphere processing, elicited a MEE for same views immediately after exposure, then for mirror views after sleep. Most importantly, sleep deprivation during the first post-exposure night, although followed by two recovery nights, extinguished MEE for all views in the LVF but not in the RVF. Besides demonstrating that post-exposure time and sleep facilitate the generalization process by which we integrate various representations of an object, our results suggest that mostly in the right hemisphere, sleep may be mandatory to consolidate the memory bias underlying affective preference. These interhemispheric differences tentatively call for a reappraisal of the role of cerebral asymmetries in wake- and sleep-dependent processes of memory consolidation.
\end{abstract}

Mere exposure effect (MEE) is obtained when incidental exposure to initially novel stimuli (e.g., nonsense words, line drawings, ideograms, faces, or novel three-dimensional objects; Bornstein 1989; Butler and Berry 2004) increases the likelihood that they will be favored over nonpresented items later on during a preference judgment. A potent explanation for the MEE is that prior encounter with a stimulus enhances its subsequent processing fluency (i.e., the speed and ease with which this stimulus is perceived), which is one of the determinants of affective preference (Reber et al. 2004). Although several other features may determine affective judgment toward the material (e.g., its intrinsic aesthetic appeal), a review of the literature suggests that processing fluency due to a prior encounter (or any other variable that facilitates fluent processing) is a main factor that can account for a preference response, especially when stimuli are emotionally neutral and unfamiliar, or responses have to be made within a short period of time (Winkielman et al. 2003; Reber et al. 2004; Willems et al. 2006).

By this fluency account, the MEE broadly falls into the same class of memory effects as priming, that is, the facilitation or bias in the processing of a stimulus as a function of a recent encounter with that stimulus (Butler and Berry 2004). Like priming (Biederman and Gerhardstein 1993), affective preference (Seamon and Delgado 1999; Lawson 2004) may generalize to stimulus transformations including depth-rotated views of pre-exposed objects. This suggests that the representation underlying enhanced fluency is abstract and viewpoint invariant, but this assumption is partially contradicted by the observation that both priming (Srinivas 1995) and mere exposure (Lawson 2004) effects may decrease or even vanish for certain rotations. Evidence gathered from priming experiments partially resolved these inconstancies

${ }^{3}$ All authors equally contributed to this study.

4Present address: Faculty of Psychology and Educational Sciences, Université Libre de Bruxelles, ULB CP191, avenue F.D. Roosevelt 50, B-1050 Bruxelles, Belgium.

${ }^{5}$ Corresponding author.

E-mail Philippe.Peigneux@ulb.ac.be; fax +32-2-650-22-09.

Article is online at http://www.learnmem.org/cgi/doi/10.1101//m.551207. in suggesting that generalization effects are subtended by hemispheric specialization in the cerebral cortex (Burgund and Marsolek 2000; Marsolek 2004). Indeed, when structural modifications are made to previously exposed stimuli, for instance objects' rotations (Burgund and Marsolek 2000), unseen prototypes of learned forms (Marsolek 1995), or different letter cases (Marsolek and Hudson 1999), performance was consistently higher for items displayed in the right visual field (RVF), which predominantly projects toward the left cerebral hemisphere (LH), than for items displayed in the left visual field (LVF), projecting toward the right hemisphere (RH). These studies have suggested that the RH is specialized for the processing of viewpointdependent, exemplar-based information, whereas the LH is better suited to process viewpoint-independent, abstract information. Accordingly, it has been shown that task and stimulus demands affect which brain hemisphere most efficiently supports priming (Burgund and Marsolek 2000) and MEE (Compton et al. 2002). Furthermore, recent neuroimaging data have confirmed the specialization of the $\mathrm{LH}$ and the $\mathrm{RH}$ for view-invariant and view-dependent processing, respectively (Koutstaal et al. 2001; Vuilleumier et al. 2002; Simons et al. 2003).

From another perspective, there is now ample evidence that post-training sleep participates in the offline processing and consolidation of recent memory traces (for reviews, see Maquet et al. 2003; Rauchs et al. 2005; Walker and Stickgold 2006). Still, the few studies having investigated a role for sleep in consolidating the memory representations subtending priming (Plihal and Born 1999; Wagner et al. 2003; Rauchs et al. 2006) or priminglike (Wagner et al. 2002) effects have yielded discrepant results. It should be noticed, however, that none of these studies has tested the effect of hemispheric specialization on the development of these memory representations. Furthermore, although it is known that priming effects can be extremely robust over extended periods of time for same exemplars (Cave 1997), it remains unknown whether post-exposure sleep plays a significant role for the generalization of priming effects toward novel instances of the exposed stimuli. Indeed, in published studies, generalization effects have always been tested immediately after ex- 

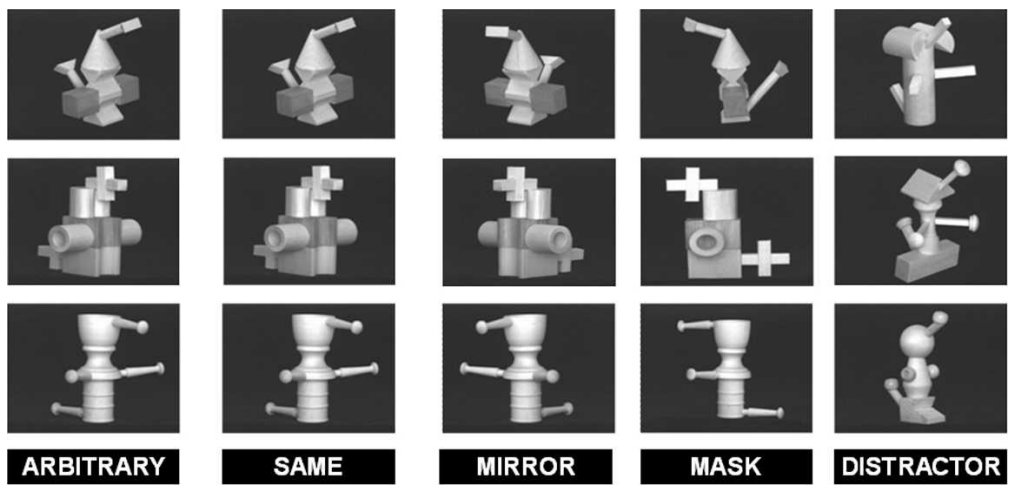

Figure 1. Sample of three-dimensional unfamiliar objects. Each row shows an object presented in its original position (ARBITRARY) during the left-right orientation decision at the exposure phase. During the liking judgment phase, objects are displayed either in the same arbitrary position (SAME), after a nearly mirror rotation (MIRROR), or after a rotation masking at least one salient part of the original object (MASK), along with non-previously seen objects (DISTRACTERS).

posure, which did not allow for assessing the influence of timedependent processes (McGaugh 1966) or a beneficial role of posttraining sleep (Maquet et al. 2003; Rauchs et al. 2005; Walker and Stickgold 2006) for the consolidation of the memory traces underlying priming or mere exposure effects.

To address these issues, we have tested whether longer time intervals including nights of sleep may contribute to the development of an affective preference toward different instances of a previously exposed stimulus. At the exposure phase, participants were presented with pictures of unfamiliar three-dimensional objects displayed in various orientations (Srinivas 1995; Seamon and Delgado 1999). They were merely asked to decide whether the object was left- or right-oriented. Afterward, they were introduced to a liking judgment phase either immediately (IMM; $n=16), 72 \mathrm{~h}$ later after three nights of regular sleep (TRS; $n=16$ ), or $72 \mathrm{~h}$ later with total sleep deprivation on the first postexposure night, followed by two recovery nights of regular sleep (TSD; $n=29$ ). Test trials (Fig. 1) were composed of previously seen objects either displayed in the same orientation (SAME) as during exposure, depth-rotated at $80^{\circ}$ in a nearly-mirror manner (MIRROR), or depth-rotated at $110^{\circ}$ in such a way that some salient parts of the original object were masked (MASK), as well as of never seen objects (distracters). Participants were instructed to decide as fast as possible whether or not they liked the presented object. Additionally, half of the items were presented in each visual hemifield during the liking judgment phase, in order to

\begin{abstract}
visual field presentation (LVF vs. RVF)
\end{abstract} and object orientation (SAME vs. MIRROR vs. MASK) factors. This analysis disclosed a main effect of visual field presentation, $F_{(1,57)}=7.7, P<0.05$, indicating that as a whole, pre-exposed stimuli were preferred to the distracters more often in the RVF (mean preference $+21 \% \pm$ standard deviation of the mean $4 \%$ ) than in the LVF $(+7 \% \pm 4 \%)$. There was also a main effect of object orientation, $F_{(2,114)}=16.7, P<0.005$. Post hoc analyses (Duncan's test) showed that on average, pre-exposed stimuli were preferred to the distracters less often in the MASK $(+2 \% \pm 4 \%)$ than in either the SAME $(+22 \% \pm 3 \%)$ or MIRROR $(+18 \% \pm 4 \%)$ orientations, $P$ s $<0.005$. The difference between SAME and MIRROR orientations was nonsignificant $(P>0.33)$.

Most importantly, we found a significant triple interaction effect, $F_{(4,114)}=4.40, P<0.005$, suggesting that the MEE was contingent upon a combination of stimulus orientation, visual field/ brain hemisphere solicited, and time elapsed between exposure and testing, sleep being allowed or not on the first post-exposure night (Fig. 2). A similar three-way ANOVA conducted on response decision times yielded only a main effect of group, $F_{(2.57)}=7.22, P s<0.005$. Duncan post-hoc tests indicated that response times were on average slower in the TSD $(1016 \pm 50$ $\mathrm{msec})$ than in the TRS $(730 \pm 66 \mathrm{msec})$ or IMM (785 $\pm 67 \mathrm{msec})$ groups $(P \mathrm{~s}<0.05)$. This effect was not attributable to attentional alterations in the TSD group at day 4 since RTs in a psychomotor vigilance task conducted immediately before the tasks were similar from exposure to test time both in TSD and TRS groups (group

Table 1. Affective preference judgment

\begin{tabular}{|c|c|c|c|c|c|c|c|c|}
\hline \multirow[b]{2}{*}{ Group } & \multirow[b]{2}{*}{ Hemisphere } & \multirow{2}{*}{$\begin{array}{c}\text { Distracter } \\
\mu(\mathrm{SEM})\end{array}$} & \multicolumn{2}{|c|}{ SAME } & \multicolumn{2}{|c|}{ MIRROR } & \multicolumn{2}{|c|}{ MASK } \\
\hline & & & $\mu(\mathrm{SEM})$ & $d$ & $\mu(\mathrm{SEM})$ & $d$ & $\boldsymbol{\mu}(\mathrm{SEM})$ & $d$ \\
\hline \multirow[t]{2}{*}{$\mathrm{IMM}$} & $\mathrm{LH}$ & $33( \pm 7)$ & $+36( \pm 8)$ & $1.86^{\star *}$ & $+30( \pm 11)$ & $1.08^{*}$ & $-5( \pm 9)$ & -0.18 \\
\hline & $\mathrm{RH}$ & $36( \pm 7)$ & $+25( \pm 9)$ & $0.79^{\star}$ & $+3( \pm 10)$ & 0.13 & $-9( \pm 11)$ & -0.40 \\
\hline \multirow[t]{2}{*}{ TRS } & $\mathrm{LH}$ & $34( \pm 4)$ & $+27( \pm 9)$ & $0.78^{*}$ & $+31( \pm 9)$ & $1.04^{* *}$ & $+23( \pm 7)$ & $1.07^{*}$ \\
\hline & $\mathrm{RH}$ & $33( \pm 4)$ & $+23( \pm 8)$ & $0.94^{*}$ & $+19( \pm 7)$ & $0.88^{*}$ & $-8( \pm 7)$ & -0.30 \\
\hline \multirow[t]{2}{*}{ TSD } & $\mathrm{LH}$ & $38( \pm 6)$ & $+21( \pm 7)$ & $0.72^{\star}$ & $+16( \pm 6)$ & $0.54^{*}$ & $+5( \pm 7)$ & 0.18 \\
\hline & $\mathrm{RH}$ & $52( \pm 6)$ & $+3( \pm 6)$ & -0.09 & $+4( \pm 6)$ & -0.10 & $+5( \pm 7)$ & -0.20 \\
\hline
\end{tabular}

Values in the "Distracter" column are mean $(\mu)$ percentages of "like" responses for the distracters presented. Values in the "SAME," "MIRROR," and "MASK" columns are mean $(\mu)$ preference scores, i.e., the percentage increase (or decrease) in "like" responses as compared with the distracters. All values are given with the standard error of the mean (SEM). Effect sizes (d) of single t-tests against the null computed on preference scores are provided with reference to the classification of effect sizes proposed by Cohen (1988); i.e., small $d=0.20$, medium $d=0.50$, and large $d=0.80$. Statistically significant tests are mentioned: $\left(^{*}\right) P<0.05,\left({ }^{* *}\right) P<0.005$.

Abbreviations: (IMM) Subjects tested immediately after exposure; (TRS) subjects tested $72 \mathrm{~h}$ after exposure including three nights of regular sleep; (TSD) subjects tested $72 \mathrm{~h}$ after exposure with sleep deprivation on the first night after exposure, followed by two recovery nights of regular sleep; (LH) left hemisphere; $(\mathrm{RH})$ right hemisphere. 
by time interaction term $\left.F_{(1,42)}=0.39, P>0.53\right)$. The addition of a gender (male vs. female) factor in the ANOVAs did not yield any supplemental effect $(P s>0.12)$, in line with a prior study (Compton et al. 2002). In the remainder of this report, we will further detail on the hemisphere by orientation effects on preference judgment scores within each group.

\section{Hemispheric asymmetries in immediate MEE}

In the IMM group, tested immediately after exposure, preference for pre-exposed objects was significant in the SAME orientation in both visual fields, and in the MIRROR orientation in the RVF only (t-tests against the null reference mean, $P$ s $<0.05$; all other t-tests $P \mathrm{~s}>0.41$; Table 1$)$. In the RVF, pre-exposed objects were preferred to the distracters more often in the SAME $(+36 \%)$ and MIRROR (+30\%) orientations than in the MASK $(-5 \%)$ orientation (Duncan's post-hoc tests, $P \mathrm{~s}<0.005)$. In the LVF, MEE was greater in the SAME $(+25 \%)$ orientation than in the MIRROR $(+3 \%)$ or MASK $(-9 \%)$ orientations (Ps $<0.05$; Fig. 2 , left). These data evidence a MEE in both cerebral hemispheres for objects displayed in the same study-test orientation, along with sensitivity of the $\mathrm{LH}$, but not of the $\mathrm{RH}$, for nearly-mirror rotations that preserved complete objects' visibility.

\section{MEE generalization with post-exposure time}

In the TRS group, tested $72 \mathrm{~h}$ after exposure with a regular sleep schedule, MEE was significant in the SAME and MIRROR orientations in both visual fields, and in the MASK orientation in the RVF only ( $P \mathrm{~s}<0.05$; no preference for MASK orientation in the LVF, $P>0.3$; Table 1). In the RVF, pre-exposed objects were equally preferred in the SAME $(+27 \%)$, MIRROR $(+31 \%)$, and MASK $(+23 \%)$ orientations $(P S>0.34)$. In the LVF, objects were preferred more in the SAME $(+23 \%)$ and MIRROR $(+19 \%)$ than in the MASK $(-8 \%)$ orientation ( $P \mathrm{~s}<0.005$; Fig. 2 , middle). These results indicate that the MEE generalizes with post-exposure time, and possibly sleep, in the RVF (LH) to different kinds of depth-rotation transformations, including those that put out of sight salient parts of the pre-exposed objects. In the LVF (RH), it

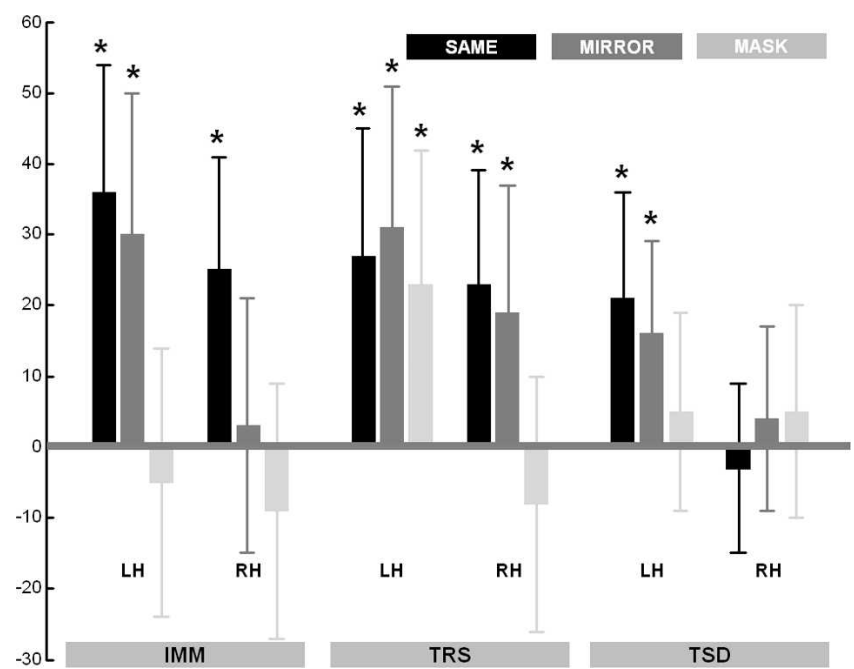

Figure 2. Preference scores for stimuli displayed in the left (LH) and right $(\mathrm{RH})$ hemispheres in SAME, MIRROR, and MASK orientations, in subjects tested immediately (IMM), $72 \mathrm{~h}$ later including three nights of regular sleep (TRS), or $72 \mathrm{~h}$ later with sleep deprivation on the first night after exposure (TSD). Bars represent the difference between the percentages of "like" responses for a given category of pre-exposed objects vs. the distracters category (thus, a zero value means no preference). (Asterisks) Significant preference score (t-test against the null reference mean): $\left.\left(^{*}\right) P<0.05,{ }^{* *}\right) P<0.005$. Error bars are standard errors of the mean. generalizes only to rotations that preserve the entire visibility of the object.

\section{Sleep, MEE generalization, and cerebral asymmetries}

Finally, we aimed specifically to probe the role of post-exposure sleep in the formation and generalization of affective preference toward pre-exposed objects. In the TSD group, sleep-deprived on the first post-exposure night then tested after two recovery nights of sleep, there was a preference in the RVF for previously displayed objects in the SAME and MIRROR (Ps $<0.05)$ orientations, but no more preference in the MASK orientation $(P>0.42)$, whereas preference was abolished in the LVF for all kinds of stimuli (PS $>0.45$; Table 1). Preference for objects presented to the RVF was higher in the SAME (+21\%) than in the MASK $(+5 \%)$ orientation $(P<0.05)$. Preference in the MIRROR $(+16 \%)$ condition was not significantly different than in the two other conditions (Ps $>0.14)$. In the LVF, no difference was found between SAME $(-3 \%)$, MIRROR $(+4 \%)$, and MASK $(+5 \%)$ orientations (Ps > 0.32; Fig. 2, right).

In the TSD condition, however, the baseline rate of "like" responses for the distracters was higher in the LVF $(52 \% \pm 5 \%)$ than in the RVF $(38 \% \pm 5 \% ; P<0.05)$, which was not the case in the IMM or TRS conditions ( $P$ s $>0.7$; Table 1$)$. Actually, this lateralized pattern of preference for distracters replicated the effects observed in a fourth group $(n=12)$, tested in a supplemental "no-exposure" condition, i.e., in volunteers who had to perform the judgment task without any prior exposure to the material. In this group, a two-way ANOVA conducted on hemisphere (LH vs. $\mathrm{RH}$ ) and stimulus type (SAME vs. MIRROR vs. MASK vs. distractor; all categories being arbitrarily defined here in the absence of a learning condition) on "like" scores disclose a main effect of hemisphere presentation $\left(F_{(1,11)}=6.84, P<0.05\right)$, actually indicating a natural propensity to produce more "like" response for items presented in the $\mathrm{RH}(57 \% \pm 5 \%)$ than in the $\mathrm{LH}$ $(45 \% \pm 4 \%)$. As expected given the lack of prior exposure to the material, interaction effect $\left(F_{(3,33)}=1.14, P>0.34\right)$ and main effect of stimulus type $(P>0.54)$ were nonsignificant. Taken together, these results indicate that in subjects deprived of sleep on the first post-training night, generalization effects are suppressed in the LH, whereas the RH behaves as if it had never gone through the original exposure session. Indeed, there was no more difference in preference judgments between any kind of exposed stimulus and the distracters in the RH.

Additionally, eye movements were recorded in the TSD and "no-exposure" conditions. Results disclosed that eye movements were made toward the displayed stimulus, both in the $\mathrm{RH}$ and in the LH (mean amplitude $6^{\circ} \pm 3^{\circ}$ of visual angle laterally in the $\mathrm{RH}$ and $6^{\circ} \pm 3^{\circ}$ in the $\mathrm{LH}$ ), suggesting that the stimuli were seen in foveal vision at some point in time. Still, there was no interhemispheric difference on the amplitude and number of saccades during the stimulus presentation, nor any interaction effects between hemisphere presentation (LH vs. RH) and object orientation (distracters, SAME, MIRROR, or mask; Ps > 0.70). Therefore, equal distribution of eye movements toward the stimulus in the LH and RH excludes a causal role of these eye movements in the observed interhemispheric or between-stimuli differences in preference judgment patterns.

\section{Discussion}

To the best of our knowledge, the present study provides the first evidence for both sleep- and laterality-dependent processes of generalization of the memory bias subtending preference judgments toward novel perspectives of previously exposed objects.

Although the number of stimuli is admittedly small within each subcondition, we have observed robust preference effects, with large effect sizes (see Table 1) for MEE comparable to previ-

\section{Learning \& Memory \\ www.learnmem.org}


ously published studies (e.g., Bornstein 1989; Willems and Van der Linden 2006). We also consistently observed hemifieldrelated differences within our population, depending on the delay and the availability of sleep on the post-training night. Therefore, we are confident that interhemifield differences in this experiment are not incidental effects. Still, the novelty of this report and the specificity of the material make that future studies are needed to draw definite conclusions about these lateralized processes of memory consolidation.

In the present study, laterality effects in preference judgments were already present at the immediate post-exposure phase, in line with the long-standing idea that cerebral asymmetries modulate cognitive specialization in the brain (e.g., Marc Dax 1836 in Springer and Deutsch 1998; Toga and Thompson 2003). Here, results for preference judgments conducted immediately after exposure disclosed a LH specialization for the generalization of the MEE. When objects were presented in the RVF, connected to the $\mathrm{LH}$, affective preference was present for same and mirrored three-dimensional objects. In the LVF, connected to the right hemisphere (RH), only objects displayed in the same orientation as in the study phase were preferred. This profile of hemispheric specialization is congruent with reports of lateralized priming effects having led to the dissociable neural subsystems theory (Marsolek 1999; Burgund and Marsolek 2000), which proposes that objects are stored in the LH in a way that produces more viewpoint-invariant effects, whereas objects are preferentially stored in the $\mathrm{RH}$ in a viewpoint-dependent manner (but see Curby et al. 2004). Additionally, competition between hemispheric-lateralized subsystems may provide an explanation for discrepant findings of viewpoint-invariant (Seamon and Delgado 1999) versus viewpoint-dependent (Lawson 2004) patterns of MEE, reported in prior studies where stimuli were displayed centrally, i.e., exposed at once to the whole visual field.

Above immediate post-exposure effects, a prominent contribution of the present study is the demonstration that MEE generalizes with post-exposure time. In the $\mathrm{LH}$, it generalizes to all types of structural transformations of unfamiliar objects, whereas in the RH it generalizes only to structural transformations where visibility on all salient parts of the object was preserved. Prior studies already showed the persistence of mere exposure (Seamon et al. 1983) or priming (Cave 1997) effects for identical (i.e., SAME) stimuli over several days or weeks. Here, we additionally demonstrate that the MEE generalizes over time toward novel transformations of the exposed objects, suggesting that the memory traces underlying fluency-based mechanisms of MEE are actively remodeled over extended periods of time up to several days. In the RH, where MEE effects were absent for mirror-rotated objects at immediate testing, an alternative interpretation would be that performance during the judgment phase was partially supported by interhemispheric transfer of abstract information consolidated in the LH (Marsolek et al. 2002). Further studies should investigate whether generalization to all types of rotations can take place in the $\mathrm{RH}$ across more extended periods of time. Also, it remains to be ascertained whether time-dependent generalization effects would be observed in the context of perceptual priming, where performance is thought to be subtended by the same memory-based processing fluency bias (Reber et al. 2004).

Considering the role of post-exposure sleep within longterm, offline processes of memory consolidation, we have found that sleep deprivation on the first night after exposure, albeit followed by two recovery nights, extinguishes affective preference for all forms of previously exposed objects in the RH. Moreover, sleep-deprived subjects exhibited a propensity to produce more "like" response for novel objects presented in the RH than in the LH, a peculiar behavior also found in subjects tested in a no-exposure condition. Combined with the abolition of preference effects, these data indicate that in the RH, subjects deprived of sleep on the first post-training night behave as if they had never gone through the original exposure session. Although far less pronounced in the $\mathrm{LH}$, the effect of sleep deprivation on the post-exposure night was also significant since the pattern of performance fell back to the effects observed for preference judgments performed immediately after exposure. Altogether, these results suggest that sleep deprivation on the night after exposure disrupts to some extent the fluency-based mechanisms of affective preference (Jacoby and Kelley 1987; Bornstein and D'Agostino 1992; Reber et al. 2004) for previously exposed objects. This would be consistent with earlier studies having shown that intervening deprivation of sleep, and especially rapid-eyemovement (REM) sleep, may alter priming (Smith 1995; Plihal and Born 1999; Wagner et al. 2003) and priming-like effects (Wagner et al. 2002). However, another study failed to disclose sleep-dependent effects using tachistoscopic identification of drawings (Rauchs et al. 2006). Along with our own data, the discrepancy between these results emphasizes the importance to assess separately the respective contributions of the left and right cerebral hemispheres in studies targeting time- and sleepdependent processes of memory consolidation. In our present study, indeed, the group by stimulus partial ANOVA conducted on preference scores irrespective of laterality presentation actually failed to reveal a significant role for post-training sleep in the development of affective preference toward previously exposed novel objects (see Additional MEE Results in Materials and Methods section). Results indicate that when data from both hemispheres are merged, SAME and MIRROR orientations are preferred above chance levels by both the TRS and TSD groups, an effect actually supported by the left hemisphere only in the TSD group. Therefore, it cannot be excluded that discrepancies between results of published studies stem from differences in the hemispheric requirements of the material and/or the experimental procedure used.

Although detrimental in both hemispheres, sleep deprivation did not affect performance to the same extent, suggesting interhemispheric differences in sleep-dependent processes of memory consolidation. Preference for SAME orientation, although present immediately after exposure, was lost in the $\mathrm{RH}$ following sleep deprivation, whereas that preference was preserved in the LH for SAME and MIRROR objects. This suggests that sleep on the night after exposure is more beneficial to the consolidation of fluency-based effects in the RH than in the LH. To the best of our knowledge, a generalization over time of affective preference or priming effects toward different instances of pre-exposed stimuli has not previously been reported. Furthermore, we reveal here that sleep-dependent effects on the consolidation of memory-based affective preference are actually prominent in the right hemisphere. From this perspective, hemispheric differences in the development of the MEE may call for a reappraisal of the role of cerebral asymmetries in wake- and sleepdependent processes of memory consolidation in humans. Nowadays, the mechanisms underlying these interhemispheric differences remain unknown. A putative candidate could be an indirect, modulatory influence of the noradrenergic systems during sleep. Indeed, noradrenergic pathways that mediate not only vigilance but also selective attention toward behaviorally relevant stimuli are more strongly lateralized in the RH (Posner and Petersen 1990; Corbetta and Shulman 2002), and it has been shown that sleep deprivation affects arousal and performance more in the RH than in the LH (Kim et al. 2001; Johnsen et al. 2002; Pallesen et al. 2004). Future studies are needed to elucidate the neural mediators underpinning laterality-based memory consolidation effects. 
Finally, it remains debatable whether MEE patterns could reflect a combination of effects of sleep on both explicit and implicit memory. On the one hand, the mere exposure effect (MEE) met the very conservative chance recognition criteria proposed by Reingold and Merikle (1990), which requires that the effect of a variable has to be shown to be greater on an implicit than on an explicit memory task. Accordingly, subliminal presentation influences more the preference judgment task than an explicit recognition memory task (e.g., Kunst-Wilson and Zajonc 1980; Bornstein 1989; Seamon et al. 1998). On the other hand, it is accepted nowadays that the processes subtending the MEE are to some extent similar to some of the processes subtending explicit recognition (e.g., familiarity/fluency based recognition; Bornstein and D'Agostino 1994; Whittlesea and Price 2001). Nonetheless, although we have not specifically tested explicit recognition in the present study, it has been previously shown that the MEE is independent of (explicit) recollection-based recognition (Bornstein 1989; Bornstein and D'Agostino 1992). Further studies should investigate whether these hemispheric effects are solely relevant to the memory traces underlying processing fluency and affective preference, especially in terms of potential implicit and explicit contributions to a sleep-dependent memory effect (Born and Wagner 2004; Robertson et al. 2004; Drosopoulos et al. 2005).

\section{Materials and Methods}

\section{Participants}

Seventy-three healthy right-handed volunteers (38 males, 35 females; mean age, 22 yr; range, 18-30 yr) gave their informed, written consent to participate in this experiment, which was approved by the Ethics Committee of the University of Liège. Subjects were explicitly instructed not to consume psychostimulants, drugs, or alcohol for $72 \mathrm{~h}$ prior to and throughout the experiment period. In the regular sleep (TRS) group (6 males, 10 females), subjects were exposed to the stimuli then allowed to sleep at home as usual for the three post-training nights. In the TSD group (16 males, 13 females), subjects were exposed to the stimuli then stayed awake in the laboratory on the first posttraining night until 7:00 a.m. During this night, participants' physical activity was maintained at as low a level as possible and subjects remained under constant supervision by the experimenters. In particular, they were instructed to remain seated throughout the experimental night and the consumption of psychoactive stimulants was prohibited. They had to engage in their usual daytime activities and slept normally at home during the next two post-training nights. Data from one subject in the sleep deprivation (TSD) group were excluded from the analyses due to alcohol consumption before the sleep deprivation night. In the TSD and TRS groups, exposure and test phases occurred at the same time of day, between 9:00 a.m. and 7:00 p.m., in order to avoid circadian confounds. In other words, each individual subject was tested at a given time of the day and then later retested at the same time of the day. In the IMM group (10 males, 6 females), subjects were exposed to the stimuli and then tested immediately afterward, between 10:00 a.m. and 5:00 p.m. In the no-learning condition ( 6 males, 6 females), subjects were tested without any prior exposure to the stimulus, between 10:00 a.m. and 6:00 p.m.

\section{Sleep, vigilance, and circadian parameters (TSD vs. TRS)}

\section{Sleep parameters}

Sleep duration and quality (from very poor [1] to very good [6]) were subjectively assessed using the St. Mary's Hospital sleep questionnaire (Ellis et al. 1981) for the one night preceding and the three nights during the study period in the TSD and TRS groups (Table 2). Mean sleep duration was not different for the TSD and TRS groups on night $1\left(t_{(29)}=-0.41, P>0.68\right)$ and night
Table 2. Sleep parameters

\begin{tabular}{|c|c|c|c|c|}
\hline & Night 1 & Night 2 & Night 3 & Night 4 \\
\hline \multicolumn{5}{|c|}{ Sleep duration (hours: mean $[ \pm S D]$ ) } \\
\hline TRS & $8.4( \pm 1.2)$ & $8.3( \pm 1.3)$ & $8.1( \pm 1.2)$ & $8.1( \pm 1.2)$ \\
\hline TSD & $8.3( \pm 1.4)$ & - & $10.1( \pm 1.7)$ & $8.4( \pm 1.4)$ \\
\hline \multicolumn{5}{|c|}{ Subjective sleep quality (rating: median [IQI]) } \\
\hline TRS & $5(1)$ & $4(1)$ & $4(1.5)$ & $4(1)$ \\
\hline TSD & $4(1.5)$ & - & $5(2)$ & $4(1)$ \\
\hline
\end{tabular}

(SD) Standard deviation of the mean; (IQI) interquartile interval; (TRS) subjects tested $72 \mathrm{~h}$ after exposure including three nights of regular sleep; (TSD) subjects tested $72 \mathrm{~h}$ after exposure with sleep deprivation on the first night after exposure, followed by two recovery nights of regular sleep.

$4\left(t_{(29)}=-1.65, P>0.1\right)$, which preceded day 1 (exposure to the stimuli) and day 4 (test session), respectively. Likewise, median subjective sleep quality was equivalent for the TSD and TRS groups for night 1 (Mann-Whitney $U=90.5, P>0.24$ ) and night $4(\mathrm{U}=94, P>0.3)$. These results suggest that all participants were tested in similar states of alertness on both day 1 and day 4 . While some subjects were sleep-deprived on night 2, the other participants slept normally at home. Finally, mean sleep duration was longer for the TSD group than for the TRS group on the night following the post-training night $\left(t_{(29)}=-3.49, P<0.005\right)$, as well as subjective sleep quality (Mann-Whitney $U=64$, $P<0.05)$, showing the expected sleep rebound after deprivation.

\section{Vigilance}

To assess psychomotor vigilance at the time of exposure and at the judgment phase in the TRS and TSD groups, subjects performed a simple reaction time task, before exposure and test phases. In this vigilance task, a white cross was repeatedly displayed in the center of a black screen after a variable time interval, randomly ranging from 2000 to $5000 \mathrm{msec}$. Subjects were instructed to press the space bar of the keyboard as fast as possible when the cross was displayed on the screen. Eighty trials were presented during one session, for an approximate duration of $6 \mathrm{~min}$. The dependent measure of vigilance was response time (RT). A two-way ANOVA conducted on RT with group (TSD vs. TRS) as between-subjects factor and time (Exposure vs. Judgment phase) as a repeated measure showed that performance was similar from exposure to test time both in subjects allowed to sleep $(357 \pm 11 \mathrm{msec}$ vs. $347 \pm 8 \mathrm{msec})$ and in subjects who were sleep deprived on the first post-exposure night $(302 \pm 11 \mathrm{msec}$ vs. $296 \pm 8 \mathrm{msec}$; group by time interaction term $F_{(1,29)}=0.03$, $P>85)$.

\section{Circadian rhythms}

A self-assessment questionnaire (Horne and Ostberg 1976) did not reveal "morningness-eveningness" differences in circadian rhythms between the TRS and TSD groups (respectively, median scores $=48$ and 50; ranges $=29-74$ and 34-68; Mann-Whitney U-test adjusted $\mathrm{Z}=-0.98, P>0.32)$.

\section{MEE task: Material, apparatus, and procedure}

Stimuli were 32 pictures of solid symmetrical objects (Srinivas 1995; Seamon and Delgado 1999). For each object, pictures were obtained in an arbitrary $0^{\circ}$ visual angle orientation (SAME), after a nearly mirror $80^{\circ}$ depth-rotated orientation that preserved visibility of all parts of the object in its arbitrary position (MIRROR), and after a $110^{\circ}$ depth-rotated orientation hiding some salient parts of the object in its arbitrary position (MASK; see Fig. 1). Illumination and black background conditions were kept constant in all orientations. Images were presented using E-Prime software (Psychology Software Tools) on a 17-inch computer screen (refresh rate $60 \mathrm{~Hz}$ ). Responses and decision times were recorded.

During the exposure (encoding) phase, 24 pictures of objects were presented twice each in randomized order. Half of the objects faced left and half faced right. Left-right view was random- 
ized across study trials. Each object was presented in its arbitrary position in the center of the screen for $2500 \mathrm{msec}$, followed by a blank screen for $3500 \mathrm{msec}$. Participants were instructed to press a key (one of two) on the computer keyboard to decide whether the object was left- or right-oriented.

During the subsequent preference judgment (test) phase, the participants were presented with test views for each of the 24 objects and eight previously unseen distracters, in a randomized order. Of the 24 pre-exposed objects, eight objects were presented in the arbitrary orientation used at encoding (SAME). Eight other objects were presented rotated at $110^{\circ}$ from the arbitrary position used at encoding, such that the visibility of all salient parts was preserved (MIRROR). The eight remaining objects were presented rotated at $110^{\circ}$ from the arbitrary position used at encoding, in such a way that at least one salient part was masked by comparison with the arbitrary encoding view (MASK). Within each category, half of the objects were presented in the left visual field (LVF) and the other half were presented in the right visual field (RVF). Hence, four objects were displayed in each experimental subcondition. With reference to the fixation point located at the center of the screen, the center of each object was positioned $7.4^{\circ}$ to the side and its inner edge was never closer than $4.2^{\circ}$. Each test trial began with the presentation of the fixation point for 750 msec. Participants were instructed to focus their attention on the fixation point and not to try to anticipate on which side of the fixation point the next object would appear. Immediately after the fixation point disappeared from view, the object was displayed on the left or right side of the screen until a response was given. Participants were asked to continue to focus their attention on the center of the screen and to decide as fast as possible whether the object was pleasant (yes or no) by pressing a key (one of two) on the computer keyboard. A blank screen was displayed for 500 msec after the subject's response, followed by the next trial. A different, counterbalanced combination of objects was assigned to pre-exposed and distracter categories for each participant. More precisely, inter-individuals counterbalancing was randomly done as well for hemispheric field presentation than between distracters, SAME, MIRROR, and MASK stimuli.

\section{Eye movements}

Presence and amplitude of eye movements were recorded using faceLAB, a stereo camera-based image processing system (www. seeingmachines.com, Seeing Machines, Inc.; sampling rate 60 $\mathrm{Hz}$; mean measure error $1^{\circ}$ of visual angle) in two subsets of the participants (13 subjects in the TSD condition and 12 subjects in the no-learning condition). Saccades were identified as variations in the position of the eye during the time of object's display, which were larger than two standard deviations from the mean position of the eye during the immediately preceding $750 \mathrm{msec}$ where the fixation cross was displayed.

\section{Additional MEE results}

The interaction between group and stimulus (i.e., irrespective of hemisphere presentation) on preference scores was significant $F_{(4,114)}=4.20, P<0.005$. Within-group Duncan's post hoc tests are as follows. In the IMM group, preference scores were higher in the SAME $(+30 \% \pm 6 \%)$ and MIRROR $(+16 \% \pm 7 \%)$ orientations than in the MASK orientation $(-7 \% \pm 7 \%), P s<0.005$. Preference for SAME was higher than for MIRROR objects $(P<0.05)$. Single t-tests against the null reference mean were significant in the SAME $(P \mathrm{~s}<0.001)$ and MIRROR (trend; $P=0.09)$ orientations, but not in the MASK orientation $(P>0.44)$. In the TRS group, preference scores were higher in the SAME (preference $+25 \% \pm 6 \%)$ and MIRROR $(+25 \% \pm 7 \%)$ orientations than in the MASK orientation $(+7 \% \pm 7 \%), P s<0.05$. Preference for SAME was not higher than for MIRROR $(P=1.0)$ objects. Single t-tests against the null reference mean were significant in the SAME and MIRROR orientations $(P S<0.005)$, but not in the MASK orientation $(P>0.16)$. In the TSD group, preference scores did not differ between the SAME $(+9 \% \pm 5 \%)$, MIRROR $(+10 \% \pm 5 \%)$, and MASK $(+5 \% \pm 5 \%)$ orientations, $P \mathrm{~s}>0.58$. Single t-tests against the null reference mean were significant in the MIRROR $(P \mathrm{~s}<0.05)$ and SAME (trend; $P=0.06)$ orientation, but not in the MASK orientation $(P>0.31)$. These data indicate that when both hemispheres are merged, post-hoc tests actually fail to reveal a significant role for post-training sleep in the development of affective preference toward previously exposed novel objects.

\section{Acknowledgments}

The study was supported by the Belgian Fonds National de la Recherche Scientifique (FNRS), the Fondation Médicale Reine Elisabeth, the Research Fund of ULg, and PAI/IAP Interuniversity Pole of Attraction P5/04. S.W. is supported by FNRS. The authors declare no competing financial interests.

\section{References}

Biederman, I. and Gerhardstein, P.C. 1993. Recognizing depth-rotated objects: Evidence for 3D viewpoint invariance. J. Exp. Psychol. Hum. Percept. Perform. 19: 1162-1182.

Born, J. and Wagner, U. 2004. Awareness in memory: Being explicit about the role of sleep. Trends Cogn. Sci. 8: 242-244.

Bornstein, R.F. 1989. Exposure and affect: Overview and meta-analysis of research, 1968-1987. Psychol. Bull. 106: 265-289.

Bornstein, R.F. and D'Agostino, P.R. 1992. Stimulus recognition and the mere exposure effect. J. Pers. Soc. Psychol. 63: 545-552.

Bornstein, R.F. and D'Agostino, P.R. 1994. The attribution and discounting of perceptual fluency: Preliminary tests of a perceptual fluency/attributional model of the mere exposure effect. Soc. Cognit. 12: $103-128$.

Burgund, E.D. and Marsolek, C.J. 2000. Viewpoint-invariant and viewpoint-dependent object recognition in dissociable neural subsystems. Psychon. Bull. Rev. 7: 480-489.

Butler, L.T. and Berry, D.C. 2004. Understanding the relationship between repetition priming and mere exposure. Br. J. Psychol. 95: $467-487$.

Cave, B.C. 1997. Very long-lasting priming in picture naming. Psychol. Sci. 8: 322-325.

Cohen, J. 1988. Statistical power analysis for the behavioral sciences, $2 \mathrm{~d}$ ed. Lawrence Erlbaum, Hillsdale, NJ.

Compton, R.J., Williamson, S., Murphy, S.G., and Heller, W. 2002. Hemispheric differences in affective response. Effect of mere exposure. Soc. Cognit. 20: 1-17.

Corbetta, M. and Shulman, G.L. 2002. Control of goal-directed and stimulus-driven attention in the brain. Nat. Rev. Neurosci. 3: 201-215.

Curby, K.M., Hayward, G., and Gauthier, I. 2004. Laterality effects in the recognition of depth-rotated novel objects. Cogn. Affect. Behav. Neurosci. 4: 100-111.

Drosopoulos, S., Wagner, U., and Born, J. 2005. Sleep enhances explicit recollection in recognition memory. Learn. Mem. 12: 44-51.

Ellis, B.W., Johns, M.W., Lancaster, R., Raptopoulos, P., Angelopoulos, N., and Priest, R.G. 1981. The St. Mary's Hospital sleep questionnaire: A study of reliability. Sleep 4: 93-97.

Gratton, G., Corballis, P.M., and Jain, S. 1997. Hemispheric organization of visual memories. J. Cogn. Neurosci. 9: 92-104.

Horne, J.A. and Ostberg, O. 1976. A self-assessment questionnaire to determine morningness-eveningness in human circadian rhythms. Int. J. Chronobiol. 4: 97-110.

Jacoby, L. and Kelley, C. 1987. Unconscious influences of memory for a prior event. Pers. Soc. Psychol. Bull. 13: 314-336.

Johnsen, B.H., Laberg, J.C., Eid, J., and Hugdahl, K. 2002. Dichotic listening and sleep deprivation: Vigilance effects. Scand. J. Psychol. 43: $413-417$.

Kim, D.J., Lee, H.P., Kim, M.S., Park, Y.J., Go, H.J., Kim, K.S., Lee, S.P., Chae, J.H., and Lee, C.T. 2001. The effect of total sleep deprivation on cognitive functions in normal adult male subjects. Int. J. Neurosci. 109: $127-137$

Koutstaal, W., Wagner, A.D., Rotte, M., Maril, A., Buckner, R.L., and Schacter, D.L. 2001. Perceptual specificity in visual object priming: Functional magnetic resonance imaging evidence for a laterality difference in fusiform cortex. Neuropsychologia 39: 184-199.

Kunst-Wilson, W.R. and Zajonc, R.B. 1980. Affective discrimination of stimuli that cannot be recognized. Science 207: 557-558.

Lawson, R. 2004. Depth rotation and mirror-image reflection reduce affective preference as well as recognition memory for pictures of novel objects. Mem. Cognit. 32: 1170-1181.

Maquet, P., Smith, C., and Stickgold, R., eds. 2003. Sleep and brain plasticity. Oxford University Press, Oxford.

Marsolek, C.J. 1995. Abstract visual-form representations in the left cerebral hemisphere. J. Exp. Psychol. Hum. Percept. Perform. 
21: $375-386$.

Marsolek, C.J. 1999. Dissociable neural subsystems underlie abstract and specific object recognition. Psychol. Sci. 10: 111-118.

Marsolek, C. 2004. Abstractionist versus exemplar-based theories of visual word priming: A subsystems resolution. Q. J. Exp. Psychol. 57: 1233-1259.

Marsolek, C.J. and Hudson, T.E. 1999. Task and stimulus demands influence letter-case-specific priming in the right cerebral hemisphere. Laterality 4: 127-147.

Marsolek, C.J., Nicholas, C.D., and Andresen, D.R. 2002. Interhemispheric communication of abstract and specific visual-form information. Neuropsychologia 40: 1983-1999.

McGaugh, J.L. 1966. Time-dependent processes in memory storage. Science 153: 1351-1358.

Pallesen, S., Johnsen, B.H., Hansen, A., Eid, J., Thayer, J.F., Olsen, T., and Hugdahl, K. 2004. Sleep deprivation and hemispheric asymmetry for facial recognition reaction time and accuracy. Percept. Mot. Skills 98: 1305-1314.

Plihal, W. and Born, J. 1999. Effects of early and late nocturnal sleep on priming and spatial memory. Psychophysiology 36: 571-582.

Posner, M.I. and Petersen, S.E. 1990. The attention system of the human brain. Annu. Rev. Neurosci. 13: 25-42.

Rauchs, G., Desgranges, B., Foret, J., and Eustache, F. 2005. The relationships between memory systems and sleep stages. J. Sleep Res. 14: $132-140$.

Rauchs, G., Lebreton, K., Bertran, F., Pélerin, A., Clochon, P., Denise, P., Foret, J., Desgranges, B., and Eustache, F. 2006. Effects of partial sleep deprivation on within- and cross-format priming. Sleep 29: $58-68$.

Reber, R., Schwarz, N., and Winkielman, P. 2004. Processing fluency and aesthetic pleasure: Is beauty in the perceiver's processing experience? Pers. Soc. Psychol. Rev. 8: 364-382.

Reingold, E.M. and Merikle, P.M. 1990. On the inter-relatedness of theory and measurement in the study of unconscious processes. Mind Lang. 5: 9-28.

Robertson, E.M., Pascual-Leone, A., and Press, D.Z. 2004. Awareness modifies the skill-learning benefits of sleep. Curr. Biol. 14: 208-212.

Seamon, J.G. and Delgado, M.R. 1999. Recognition memory and affective preference for depth-rotated solid objects: Part-based structural descriptions may underlie the mere exposure effect. Vis. Cognit. 6: 145-164.

Seamon, J.G., Brody, N., and Kauff, D.M. 1983. Affective discrimination of stimuli that are not recognized: II. Effect of delay between study and test. Bull. Psychon. Soc. 21: 187-189.
Seamon, J.G., McKenna, P.A., and Binder, N. 1998. The mere exposure effect is differentially sensitive to different judgment tasks. Conscious. Cognit. 7: 85-102.

Simons, J.S., Koutstaal, W., Prince, S., Wagner, A.D., and Schacter, D.L. 2003. Neural mechanisms of visual object priming: Evidence for perceptual and semantic distinctions in fusiform cortex. Neuroimage 19: 613-626.

Smith, C. 1995. Sleep states and memory processes. Behav. Brain Res. 69: $137-145$.

Springer, S. and Deutsch, G. 1998. Left brain, right brain: Perspectives from cognitive neuroscience. Freeman, New York.

Srinivas, K. 1995. Representation of rotated objects in explicit and implicit memory. J. Exp. Psychol. Learn. Mem. Cognit. 21: 1019-1036.

Toga, A.W. and Thompson, P.M. 2003. Mapping brain asymmetry. Nat. Rev. Neurosci. 4: 37-48.

Vuilleumier, P., Henson, R.N., Driver, J., and Dolan, R.J. 2002. Multiple levels of visual object constancy revealed by event-related fMRI of repetition priming. Nat. Neurosci. 5: 491-499.

Wagner, U., Fischer, S., and Born, J. 2002. Changes in emotional responses to aversive pictures across periods rich in slow-wave sleep versus rapid eye movement sleep. Psychosom. Med. 64: 627-634.

Wagner, U., Hallschmid, M., Verleger, R., and Born, J. 2003. Signs of REM sleep dependent enhancement of implicit face memory: A repetition priming study. Biol. Psychol. 62: 197-210.

Walker, M.P. and Stickgold, R. 2006. Sleep, memory, and plasticity. Annu. Rev. Psychol. 57: 139-166.

Whittlesea, B.W. and Price, J.R. 2001. Implicit/explicit memory versus analytic/nonanalytic processing: Rethinking the mere exposure effect. Mem. Cognit. 29: 234-246.

Winkielman, P., Schwarz, N., Fazendeiro, T., and Reber, R. 2003. The hedonic marking of processing fluency: Implications for evaluative judgment. In The psychology of evaluation: Affective processes in cognition and emotion. (eds. J. Musch and K.C. Klauer), pp. 189-217. Lawrence Erlbaum, Mahwah, NJ.

Willems, S. and Van der Linden, M. 2006. Mere exposure effect: A consequence of direct and indirect fluency-preference links. Conscious. Cogn. 15: 323-341.

Willems, S., Van der Linden, M., and Bastin, C. 2006. The contribution of processing fluency to preference: A comparison with familiarity-based recognition. Eur. J. Cognit. Psychol. 19: 119-140.

Received February 9, 2007; accepted in revised form April 30, 2007. 


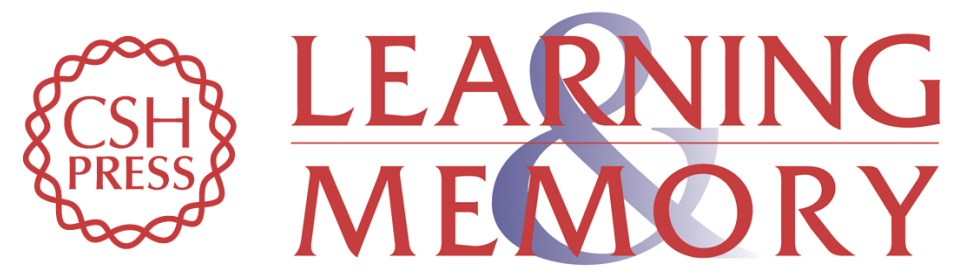

\section{Cerebral asymmetries in sleep-dependent processes of memory consolidation}

Philippe Peigneux, Remy Schmitz and Sylvie Willems

Learn. Mem. 2007, 14:

Access the most recent version at doi:10.1101//m.551207

References This article cites 49 articles, 4 of which can be accessed free at: http://learnmem.cshlp.org/content/14/6/400.full.html\#ref-list-1

License

Email Alerting Receive free email alerts when new articles cite this article - sign up in the box at the Service top right corner of the article or click here. 